\title{
THE STEINBERG-LUSZTIG TENSOR PRODUCT THEOREM, CASSELMAN-SHALIKA, AND LLT POLYNOMIALS
}

\author{
MARTINA LANINI AND ARUN RAM \\ Dedicated to Friedrich Knop and Peter Littelmann on the occasion of their 60th birthdays
}

\begin{abstract}
In this paper we establish a Steinberg-Lusztig tensor product theorem for abstract Fock space. This is a generalization of the type A result of Leclerc-Thibon and a Grothendieck group version of the Steinberg-Lusztig tensor product theorem for representations of quantum groups at roots of unity. Although the statement can be phrased in terms of parabolic affine Kazhdan-Lusztig polynomials and thus has geometric content, our proof is combinatorial, using the theory of crystals (Littelmann paths). We derive the Casselman-Shalika formula as a consequence of the Steinberg-Lusztig tensor product theorem for abstract Fock space.
\end{abstract}

\section{INTRODUCTION}

In our previous paper with P. Sobaje LRS we provided a construction of an "abstract" Fock space $\mathcal{F}_{\ell}$ in a general Lie-type setting. The construction is given by simple combinatorial "straightening relations" which generalize the KashiwaraMiwa-Stern [KMS formulation of the $q$-Fock space from the type A case. We showed that the abstract Fock space is a combinatorial realization of the graded Grothendieck group of finite dimensional representations of the quantum group at a root of unity, where the standard basis elements $|\lambda\rangle$ correspond to the Weyl modules $\Delta_{q}(\lambda)$ and the KL-basis $C_{\lambda}$ corresponds to the simple modules $L_{q}(\lambda)$. The KL-basis $C_{\lambda}$ for abstract Fock space $\mathcal{F}_{\ell}$ is an analogue of the Kazhdan-Lusztig basis (KL-basis) of the Iwahori-Hecke algebra.

In Section 1 we prove a product theorem (Theorem 1.4) in abstract Fock space which generalizes the type A theorem of Leclerc and Thibon [LT, Theorem 6.9]. Our proof follows the same pattern as the proof for type A given in [LT, Theorem 6.9] except that, in order to deal with general Lie-type, we have replaced the use of ribbon tableaux with the crystal basis and Littelmann paths. The basic philosophy of our technique is similar to the main idea of a paper of Guilhot $\mathrm{Gu}$. but we also make use of the elegant cancellation technique of Littelmann Li, proof of Theorem 9.1] to complete the proof. This technique provides a combinatorial control of the Demazure operator used in the proof of [Knp, Lemma 4.4]. We have not considered the unequal parameter case in this paper but the close relation between our context

Received by the editors April 15, 2018, and, in revised form, January 31, 2019.

2010 Mathematics Subject Classification. Primary 17B37; Secondary 20C20.

Key words and phrases. Quantum groups, affine Lie algebras, Hecke algebras, symmetric functions.

This research was partially supported by grants DP1201001942 and DP130100674.

The first author was partially supported by Australian Research Council grant DP150103525. The first author also acknowledges the MIUR Excellence Department Project awarded to the Department of Mathematics, University of Rome Tor Vergata, CUP E83C18000100006. 
and that of $\mathrm{Knp}$ cries out for an extension of the tensor product theorem for abstract Fock space to unequal parameters.

The Casselman-Shalika formula is important in the representation theory of $p$ adic groups (see [CS]), in its relation to the affine Hecke algebra (see for example $[\mathrm{BBF}]$ ) and in the geometric Langlands program (see [FGV] and [NP]). In Section 2 we show that the Casselman-Shalika formula can be derived as a special case of the Steinberg tensor product theorem for abstract Fock space. This derivation is done by using the relationship between the abstract Fock space and the affine Hecke algebra as detailed in [LRS, Theorem 4.7].

In Section 3, we review the connection between the abstract Fock space and the representations of quantum groups at roots of unity (Theorem 3.1) and the Steinberg-Lusztig tensor product theorem (Theorem 3.2). The Steinberg-Lusztig tensor product theorem is the primary motivation for the product theorem in abstract Fock space. Our approach does provide an alternative proof of the SteinbergLusztig tensor product theorem for representations of quantum groups at roots of unity (though hardly elementary since proving the Steinberg-Lusztig tensor product theorem this way relies on deep results of Kazhdan-Lusztig [KL94 and KashiwaraTanisaki [KT95]).

As explained in [LT, the Steinberg-Lustzig tensor product theorem and the abstract Fock space are intimately related to the LLT polynomials defined in type A by Lascoux, Leclerc, and Thibon [LLT. Fundamentally, the LLT polynomials are taking the role of the characters of the Frobenius twisted Weyl modules which, by the Steinberg-Lusztig tensor product theorem, are simple modules for the quantum group at a root of unity. General Lie-type definitions of LLT polynomials have been given by Grojnowski-Haiman [GH] and Lecouvey Lcy. In the second half of Section 3, we summarize a 2008 letter from C. Lecouvey to A. Ram which explains that a consequence of the tensor product theorem for abstract Fock space is that the definition from [GH] and the definition from Lcy coincide up to a power of $t^{\frac{1}{2}}$.

Kazhdan and Lusztig [KL94 established an equivalence of categories between an appropriate category of representations of the affine Lie algebra (of negative level) and the finite dimensional representations of the quantum group (of the finite dimensional Lie algebra) at a root of unity. In Section 4 we review this correspondence and make explicit the tensor product theorem in terms of representations of the affine Lie algebra. This produces a character formula for certain negative level irreducible highest weight representations of the affine Lie algebra. From the point of view of this paper this character formula is an easy consequence of [KL94] and [Lu89. We find it difficult to believe that this formula has not been noticed before but we have not yet been able to locate a suitable specific reference.

\section{A product theorem in abstract Fock SPACE $\mathcal{F}_{\ell}$}

Let $W_{0}$ be a finite Weyl group, generated by simple reflections $s_{1}, \ldots, s_{n}$, and acting on a lattice of weights $\mathfrak{a}_{\mathbb{Z}}^{*}$. For example, this situation arises when $T$ is a maximal torus of a reductive algebraic group $G$,

$$
\mathfrak{a}_{\mathbb{Z}}^{*}=\operatorname{Hom}\left(T, \mathbb{C}^{\times}\right) \quad \text { and } \quad W_{0}=N(T) / T,
$$

where $N(T)$ is the normalizer of $T$ in $G$. The simple reflections in $W_{0}$ correspond to a choice of Borel subgroup $B$ of $G$ which contains $T$. Let $R^{+}$denote the positive roots. Let $\alpha_{1}, \ldots, \alpha_{n}$ be the simple roots and let $\alpha_{1}^{\vee}, \ldots, \alpha_{n}^{\vee}$ be the simple coroots. 
The dot action of $W_{0}$ on $\mathfrak{a}_{\mathbb{Z}}^{*}$ is given by

$$
w \circ \lambda=w(\lambda+\rho)-\rho, \quad \text { where } \quad \rho=\frac{1}{2} \sum_{\alpha \in R^{+}} \alpha
$$

is the half sum of the positive roots for $G$ (with respect to $B$ ).

Fix $\ell \in \mathbb{Z}_{>0}$. The abstract Fock space $\mathcal{F}_{\ell}$ is the $\mathbb{Z}\left[t^{\frac{1}{2}}, t^{-\frac{1}{2}}\right]$-module generated by $\left\{|\lambda\rangle \mid \lambda \in \mathfrak{a}_{\mathbb{Z}}^{*}\right\}$ with relations

$$
\left|s_{i} \circ \lambda\right\rangle= \begin{cases}-|\lambda\rangle & \text { if }\left\langle\lambda+\rho, \alpha_{i}^{\vee}\right\rangle \in \ell \mathbb{Z}_{\geq 0}, \\ -t^{\frac{1}{2}}|\lambda\rangle & \text { if } 0<\left\langle\lambda+\rho, \alpha_{i}^{\vee}\right\rangle<\ell, \\ -t^{\frac{1}{2}}\left|s_{i} \circ \lambda^{(1)}\right\rangle-\left|\lambda^{(1)}\right\rangle-t^{\frac{1}{2}}|\lambda\rangle & \text { if }\left\langle\lambda+\rho, \alpha_{i}^{\vee}\right\rangle>\ell \text { and }\left\langle\lambda+\rho, \alpha_{i}^{\vee}\right\rangle \notin \ell \mathbb{Z},\end{cases}
$$

where $\lambda^{(1)}=\lambda-j \alpha_{i}$ if $\left\langle\lambda+\rho, \alpha_{i}^{\vee}\right\rangle=k \ell+j$ with $k \in \mathbb{Z}_{>0}$ and $j \in\{1, \ldots, \ell-1\}$. Note that these relations are specified only for weights $\lambda$ such that $\left\langle s_{i} \circ \lambda, \alpha_{i}^{\vee}\right\rangle<0$.

Figure 1 illustrates the terms in (1.3). This is the case $G=S L_{2}$ with $\ell=5$, $\left\langle\omega_{1}, \alpha_{1}^{\vee}\right\rangle=1$, and $\alpha_{1}=2 \omega_{1}$ and, in the figure, $\lambda$ corresponds to the third case of (1.3), $\mu$ to the first case, and $\nu$ to the second case.

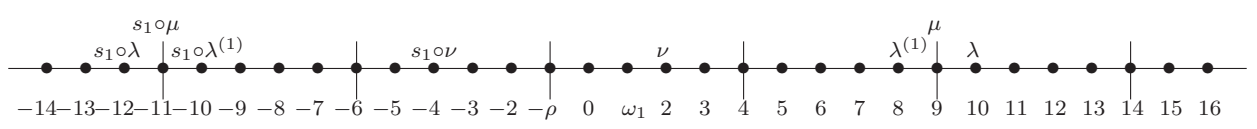

Figure 1

Define a $\mathbb{Z}$-linear involution ${ }^{-}: \mathcal{F}_{\ell} \rightarrow \mathcal{F}_{\ell}$ by

$$
\overline{t^{\frac{1}{2}}}=t^{-\frac{1}{2}} \quad \text { and } \quad \overline{|\lambda\rangle}=(-1)^{\ell\left(w_{0}\right)}\left(t^{-\frac{1}{2}}\right)^{\ell\left(w_{0}\right)-N_{\lambda}}\left|w_{0} \circ \lambda\right\rangle .
$$

where $w_{0}$ is the longest element of $W_{0}, \ell\left(w_{0}\right)=\operatorname{Card}\left(R^{+}\right)$is the length of $w_{0}$, and $N_{\lambda}=\operatorname{Card}\left\{\alpha \in R^{+} \mid\left\langle\lambda+\rho, \alpha^{\vee}\right\rangle \in \ell \mathbb{Z}\right\}$.

The dominant integral weights with the dominance partial order $\leq$ are the elements of

$$
\begin{gathered}
\left(\mathfrak{a}_{\mathbb{Z}}^{*}\right)^{+}=\left\{\lambda \in \mathfrak{a}_{\mathbb{Z}}^{*} \mid\left\langle\lambda+\rho, \alpha_{i}^{\vee}\right\rangle>0 \text { for } i=1,2, \ldots, n\right\} \\
\text { with } \quad \mu \leq \lambda \quad \text { if } \quad \mu \in \lambda-\sum_{\alpha \in R^{+}} \mathbb{Z}_{\geq 0} \alpha .
\end{gathered}
$$

In [LRS, Theorem 1.1 and Proposition 2.1] we showed that $\mathcal{F}_{\ell}$ has bases

$$
\left\{|\lambda\rangle \mid \lambda \in\left(\mathfrak{a}_{\mathbb{Z}}^{*}\right)^{+}\right\} \quad \text { and } \quad\left\{C_{\lambda} \mid \lambda \in\left(\mathfrak{a}_{\mathbb{Z}}^{*}\right)^{+}\right\}
$$

where $C_{\lambda}$ are determined by

$$
\overline{C_{\lambda}}=C_{\lambda} \quad \text { and } \quad C_{\lambda}=|\lambda\rangle+\sum_{\mu \neq \lambda} p_{\mu \lambda}|\mu\rangle \quad \text { with } p_{\mu \lambda} \in t^{\frac{1}{2}} \mathbb{Z}\left[t^{\frac{1}{2}}\right] .
$$

1.1. The action of $\mathbb{K}[X]^{W_{0}}$ on $\mathcal{F}_{\ell}$. Letting $\mathbb{K}=\mathbb{Z}\left[t^{\frac{1}{2}}, t^{-\frac{1}{2}}\right]$, the group algebra of $\mathfrak{a}_{\mathbb{Z}}^{*}$ is

$$
\mathbb{K}[X]=\mathbb{K}-\operatorname{span}\left\{X^{\mu} \mid \mu \in \mathfrak{a}_{\mathbb{Z}}^{*}\right\} \quad \text { with } \quad X^{\mu} X^{\nu}=X^{\mu+\nu}
$$


The Weyl group $W_{0}$ acts $\mathbb{K}$-linearly on $\mathbb{K}[X]$ by

(1.9)

$$
w X^{\mu}=X^{w \mu} \text { for } w \in W_{0} \text { and } \mu \in \mathfrak{a}_{\mathbb{Z}}^{*}, \quad \text { and } \quad \mathbb{K}[X]^{W_{0}}=\{f \in \mathbb{K}[X] \mid w f=f\}
$$

is the ring of symmetric functions.

Let $V$ be the free $\mathbb{K}$-module generated by $\left\{|\lambda\rangle \mid \lambda \in \mathfrak{a}_{\mathbb{Z}}^{*}\right\}$ so that

$$
\mathcal{F}_{\ell} \cong V / I
$$

where $I$ is the subspace of $V$ consisting of $\mathbb{K}$-linear combinations of the elements

$$
\begin{array}{cl}
a_{\lambda}=\left|s_{i} \circ \lambda\right\rangle+|\lambda\rangle & \text { with }\left\langle\lambda+\rho, \alpha_{i}^{\vee}\right\rangle \in \ell \mathbb{Z}_{\geq 0}, \\
b_{\lambda}=\left|s_{i} \circ \lambda\right\rangle+t^{\frac{1}{2}}|\lambda\rangle & \text { with } 0<\left\langle\lambda+\rho, \alpha_{i}^{\vee}\right\rangle<\ell, \text { and } \\
c_{\lambda}=\left|s_{i} \circ \lambda\right\rangle+t^{\frac{1}{2}}\left|s_{i} \circ \lambda^{(1)}\right\rangle+\left|\lambda^{(1)}\right\rangle+t^{\frac{1}{2}}|\lambda\rangle & \text { with }\left\langle\lambda+\rho, \alpha_{i}^{\vee}\right\rangle \ell \ell \\
& \text { and }\left\langle\lambda+\rho, \alpha_{i}^{\vee}\right\rangle \notin \ell \mathbb{Z} .
\end{array}
$$

Let $\mathfrak{g}$ be the Lie algebra of the reductive group $G$ alluded to in (1.1). Let $\varphi$ be the highest weight of the adjoint representation and let $\varphi^{\vee} \in\left[\mathfrak{g}_{\varphi}, \mathfrak{g}_{-\varphi}\right]$ such that $\left\langle\varphi, \varphi^{\vee}\right\rangle=2$ (so that $\varphi^{\vee}$ is an appropriate normalized highest short coroot of $\mathfrak{g}$ ).

$$
\text { The dual Coxeter number is } \quad h=\left\langle\rho, \varphi^{\vee}\right\rangle+1 \text {. }
$$

The level $(-\ell-h)$ action of $\mathbb{K}[X]$ on $V$ is the $\mathbb{K}$-linear extension of

$$
X^{\mu} \cdot|\gamma\rangle=\left|-\ell w_{0} \mu+\gamma\right\rangle \quad \text { for } \mu, \gamma \in \mathfrak{a}_{\mathbb{Z}}^{*}
$$

(for explanation of the terminology "level $(-\ell-h)$ dot action" and the reason that $h$ does not appear in this formula see Remark 1.3). Letting $w_{0}$ be the longest element of $W_{0}$, define

$$
\mu^{*}=-w_{0} \mu \quad \text { and } \quad w^{*}=w_{0} w w_{0} \quad \text { for } \mu \in \mathfrak{a}_{\mathbb{Z}}^{*} \text { and } w \in W_{0} .
$$

This notation is such that if $\mu \in \mathfrak{a}_{\mathbb{Z}}^{*}$ and $L_{\mathfrak{g}}(\mu)$ denotes the irreducible $\mathfrak{\mathfrak { g }}$-module of highest weight $\mu$, then the dual $L_{\mathfrak{g}}(\mu)^{*} \cong L_{\mathfrak{g}}\left(\mu^{*}\right)$. Then

$$
X^{\mu} \cdot|\gamma\rangle=\left|\ell \mu^{*}+\gamma\right\rangle \quad \text { and } \quad(w \mu)^{*}=w^{*} \mu^{*} .
$$

The following proposition establishes an action of the ring of symmetric functions $\mathbb{K}[X]^{W_{0}}$ on the abstract Fock space $\mathcal{F}_{\ell}$. From the point of view of Theorem 2.2 below, this action is coming from an action of the center of the affine Hecke algebra which, by an important result of Bernstein, is the ring of symmetric functions (inside the affine Hecke algebra). Our proof of Proposition 1.1 provides an independent proof of the existence of the action of $\mathbb{K}[X]^{W_{0}}$ without referring to the affine Hecke algebra and the characterization of its center.

Proposition 1.1. The action of $\mathbb{K}[X]$ on $V$ given in (1.12) induces a $\mathbb{K}$-linear action of the ring $\mathbb{K}[X]^{W_{0}}$ of symmetric functions on $\mathcal{F}_{\ell}$ by

$$
\left(\sum_{w \in W_{0}} X^{w \mu}\right) \cdot|\gamma\rangle=\sum_{w \in W_{0}}\left|\ell(w \mu)^{*}+\gamma\right\rangle \quad \text { for } \mu \in \mathfrak{a}_{\mathbb{Z}}^{*} \text { and } \gamma \in \mathfrak{a}_{\mathbb{Z}}^{*} .
$$


Proof. Let $f$ be an element of the subspace $I$ defined in (1.10), let $\mu \in \mathfrak{a}_{\mathbb{Z}}^{*}$, and let $i \in\{1, \ldots, n\}$. Summing over a set of representatives of the cosets in $\left\{1, s_{i}^{*}\right\} \backslash W_{0}$,

$$
\left(\sum_{w \in W_{0}} X^{w \mu}\right) \cdot f=\left(\sum_{v \in\left\{1, s_{i}^{*}\right\} \backslash W_{0}}\left(X^{v \mu}+X^{s_{i}^{*} v \mu}\right)\right) \cdot f
$$

where the representatives $v \in\left\{1, s_{i}^{*}\right\} \backslash W_{0}$ are chosen such that $\left\langle v \mu, \alpha_{i^{*}}^{\vee}\right\rangle \in \mathbb{Z}_{\geq 0}$.

Case 1. $f=\left|s_{i} \circ \lambda\right\rangle+|\lambda\rangle$ with $\left\langle\lambda+\rho, \alpha_{i}^{\vee}\right\rangle \in \ell \mathbb{Z}_{\geq 0}$. Then

$$
\begin{aligned}
\left(X^{s_{i}^{*} v \mu}\right. & \left.+X^{v \mu}\right) \cdot\left(\left|s_{i} \circ \lambda\right\rangle+|\lambda\rangle\right) \\
& =\left|\ell\left(s_{i}^{*} v \mu\right)^{*}+s_{i} \circ \lambda\right\rangle+\left|\ell(v \mu)^{*}+s_{i} \circ \lambda\right\rangle+\left|\ell\left(s_{i}^{*} v \mu\right)^{*}+\lambda\right\rangle+\left|\ell(v \mu)^{*}+\lambda\right\rangle \\
& =\left|\ell s_{i} v^{*} \mu^{*}+s_{i} \circ \lambda\right\rangle+\left|\ell v^{*} \mu^{*}+s_{i} \circ \lambda\right\rangle+\left|\ell s_{i} v^{*} \mu^{*}+\lambda\right\rangle+\left|\ell v^{*} \mu^{*}+\lambda\right\rangle \\
& =\left|s_{i} \circ\left(\ell v^{*} \mu^{*}+\lambda\right)\right\rangle+\left|s_{i} \circ\left(\ell s_{i} v^{*} \mu^{*}+\lambda\right)\right\rangle+\left|\ell s_{i} v^{*} \mu^{*}+\lambda\right\rangle+\left|\ell v^{*} \mu^{*}+\lambda\right\rangle \\
& = \begin{cases}a_{\ell v^{*} \mu^{*}+s_{i} \circ \lambda}+a_{\ell v^{*} \mu^{*}+\lambda} & \text { if }\left\langle\ell v^{*} \mu^{*}, \alpha_{i}^{\vee}\right\rangle>\left\langle\lambda+\rho, \alpha_{i}^{\vee}\right\rangle, \\
a_{\ell s_{i} v^{*} \mu^{*}+\lambda}+a_{\ell v^{*} \mu^{*}+\lambda} & \text { if }\left\langle\ell v^{*} \mu^{*}, \alpha_{i}^{\vee}\right\rangle \leq\left\langle\lambda+\rho, \alpha_{i}^{\vee}\right\rangle .\end{cases}
\end{aligned}
$$

Thus the right-hand side is an element of $I$.

Case 2. $f=\left|s_{i} \circ \lambda\right\rangle+t^{\frac{1}{2}}|\lambda\rangle$ with $0<\left\langle\lambda+\rho, \alpha_{i}^{\vee}\right\rangle<\ell$. Then $\ell v^{*} \mu^{*}+s_{i} \circ \lambda=$ $\left(\ell v^{*} \mu^{*}+\lambda\right)^{(1)}$ so that

$$
\begin{aligned}
\left(X^{s_{i}^{*} v \mu}\right. & \left.+X^{v \mu}\right) \cdot\left(\left|s_{i} \circ \lambda\right\rangle+t^{\frac{1}{2}}|\lambda\rangle\right) \\
& =\left|\ell\left(s_{i}^{*} v \mu\right)^{*}+s_{i} \circ \lambda\right\rangle+\left|\ell(v \mu)^{*}+s_{i} \circ \lambda\right\rangle+t^{\frac{1}{2}}\left|\ell\left(s_{i}^{*} v \mu\right)^{*}+\lambda\right\rangle+t^{\frac{1}{2}}\left|\ell(v \mu)^{*}+\lambda\right\rangle \\
& =\left|\ell s_{i} v^{*} \mu^{*}+s_{i} \circ \lambda\right\rangle+\left|\ell v^{*} \mu^{*}+s_{i} \circ \lambda\right\rangle+t^{\frac{1}{2}}\left|\ell s_{i} v^{*} \mu^{*}+\lambda\right\rangle+t^{\frac{1}{2}}\left|\ell v^{*} \mu^{*}+\lambda\right\rangle \\
& =\left|s_{i} \circ\left(\ell v^{*} \mu^{*}+\lambda\right)\right\rangle+\left|\left(\ell v^{*} \mu^{*}+\lambda\right)^{(1)}\right\rangle+t^{\frac{1}{2}}\left|s_{i} \circ\left(\ell v^{*} \mu^{*}+s_{i} \circ \lambda\right)\right\rangle+t^{\frac{1}{2}}\left|\ell v^{*} \mu^{*}+\lambda\right\rangle \\
& =\left|s_{i} \circ\left(\ell v^{*} \mu^{*}+\lambda\right)\right\rangle+t^{\frac{1}{2}}\left|s_{i} \circ\left(\ell v^{*} \mu^{*}+\lambda\right)^{(1)}\right\rangle+\left|\left(\ell v^{*} \mu^{*}+\lambda\right)^{(1)}\right\rangle+t^{\frac{1}{2}}\left|\ell v^{*} \mu^{*}+\lambda\right\rangle \\
& = \begin{cases}c_{\ell v^{*} \mu^{*}+\lambda} & \text { if }\left\langle v^{*} \mu^{*}, \alpha_{i}^{\vee}\right\rangle \in \mathbb{Z}_{>0}, \\
2 b_{\ell v^{*} \mu^{*}+\lambda} & \text { if }\left\langle v^{*} \mu^{*}, \alpha_{i}^{\vee}\right\rangle=0,\end{cases}
\end{aligned}
$$

since if $s_{i}^{*} v \mu \neq v \mu$, then $s_{i} v^{*} \mu^{*} \neq v^{*} \mu^{*}$ and $\left\langle v^{*} \mu^{*}, \alpha_{i}^{\vee}\right\rangle \in \mathbb{Z}_{>0}$, then $\left\langle\ell v^{*} \mu^{*}+\lambda+\right.$ $\left.\rho, \alpha_{i}^{\vee}\right\rangle>\ell$ and $\left\langle\ell v^{*} \mu^{*}+\lambda+\rho, \alpha_{i}^{\vee}\right\rangle \notin \ell \mathbb{Z}$. Thus the right-hand side is an element of $I$.

Case 3. Assume $\lambda \in \mathfrak{a}_{\mathbb{Z}}^{*}$ with $\left\langle\lambda+\rho, \alpha_{i}^{\vee}\right\rangle>\ell$ and $\left\langle\lambda+\rho, \alpha_{i}^{\vee}\right\rangle \notin \ell \mathbb{Z}$. If $\mu \in \mathfrak{a}_{\mathbb{Z}}^{*}$ and $\left\langle\nu, \alpha_{i}^{*}\right\rangle \in \mathbb{Z}_{\geq 0}$, then

$s_{i} \circ\left(v^{*} \mu^{*}+\nu\right)=s_{i}\left(v^{*} \mu^{*}+\nu+\rho\right)-\rho=s_{i} v^{*} \mu^{*}+s_{i} \circ \nu$ and $\left(\ell \nu^{*}+\lambda\right)^{(1)}=\ell \nu^{*}+\lambda^{(1)}$, 
so that, with $\left\langle\lambda+\rho, \alpha_{i}^{\vee}\right\rangle=k \ell+j$ with $k>0$ and $0 \leq j<\ell$,

$$
\begin{aligned}
& \left(X^{s_{i}^{*} v \mu}+X^{v \mu}\right) \cdot\left(\left|s_{i} \circ \lambda\right\rangle+t^{\frac{1}{2}}\left|s_{i} \circ \lambda^{(1)}\right\rangle+\left|\lambda^{(1)}\right\rangle+t^{\frac{1}{2}}|\lambda\rangle\right) \\
& =\left|\ell\left(s_{i}^{*} v \mu\right)^{*}+s_{i} \circ \lambda\right\rangle+\left|\ell(v \mu)^{*}+s_{i} \circ \lambda\right\rangle+t^{\frac{1}{2}}\left|\ell\left(s_{i}^{*} v \mu\right)^{*}+s_{i} \circ \lambda^{(1)}\right\rangle \\
& +t^{\frac{1}{2}}\left|\ell(v \mu)^{*}+s_{i} \circ \lambda^{(1)}\right\rangle \\
& +\left|\ell\left(s_{i}^{*} v \mu\right)^{*}+\lambda^{(1)}\right\rangle+\left|\ell(v \mu)^{*}+\lambda^{(1)}\right\rangle+t^{\frac{1}{2}}\left|\ell\left(s_{i}^{*} v \mu\right)^{*}+\lambda\right\rangle+t^{\frac{1}{2}}\left|\ell(v \mu)^{*}+\lambda\right\rangle \\
& =\left|\ell s_{i} v^{*} \mu^{*}+s_{i} \circ \lambda\right\rangle+\left|\ell v^{*} \mu^{*}+s_{i} \circ \lambda\right\rangle+t^{\frac{1}{2}}\left|\ell s_{i} v^{*} \mu^{*}+s_{i} \circ \lambda^{(1)}\right\rangle \\
& +t^{\frac{1}{2}}\left|\ell v^{*} \mu^{*}+s_{i} \circ \lambda^{(1)}\right\rangle \\
& +\left|\ell s_{i} v^{*} \mu^{*}+\lambda^{(1)}\right\rangle+\left|\ell v^{*} \mu^{*}+\lambda^{(1)}\right\rangle+t^{\frac{1}{2}}\left|\ell s_{i} v^{*} \mu^{*}+\lambda\right\rangle+t^{\frac{1}{2}}\left|\ell v^{*} \mu^{*}+\lambda\right\rangle \\
& =\left|s_{i} \circ\left(\ell v^{*} \mu^{*}+\lambda\right)\right\rangle+\left|s_{i} \circ\left(\ell s_{i} v^{*} \mu^{*}+\lambda\right)\right\rangle+t^{\frac{1}{2}}\left|s_{i} \circ\left(\ell v^{*} \mu^{*}+\lambda^{(1)}\right)\right\rangle \\
& +t^{\frac{1}{2}}\left|s_{i} \circ\left(\ell s_{i} v^{*} \mu^{*}+\lambda^{(1)}\right)\right\rangle \\
& +\left|\ell s_{i} v^{*} \mu^{*}+\lambda^{(1)}\right\rangle+\left|\left(\ell v^{*} \mu^{*}+\lambda\right)^{(1)}\right\rangle+t^{\frac{1}{2}}\left|\ell s_{i} v^{*} \mu^{*}+\lambda\right\rangle+t^{\frac{1}{2}}\left|\ell v^{*} \mu^{*}+\lambda\right\rangle \\
& = \begin{cases}c_{\lambda+\ell v^{*} \mu^{*}}+c_{s_{i} \circ \lambda(1)}+v^{*} \mu^{*} & \text { if }\left\langle\ell v^{*} \mu^{*}, \alpha_{i}^{\vee}\right\rangle>\ell k>0, \\
c_{\lambda+\ell v^{*} \mu^{*}}+c_{\lambda+s_{i} v^{*} \mu^{*}} & \text { if } 0<\left\langle\ell v^{*} \mu^{*}, \alpha_{i}^{\vee}\right\rangle<\ell k, \\
c_{\lambda+\ell v^{*} \mu^{*}}+b_{\lambda+s_{i} v^{*} \mu^{*}}+b_{s_{i} \circ \lambda(1)}+v^{*} \mu^{*} & \text { if }\left\langle\ell v^{*} \mu^{*}, \alpha_{i}^{\vee}\right\rangle=\ell k .\end{cases}
\end{aligned}
$$

Thus the right-hand side is an element of $I$.

These computations show that $I$ is stable under the action of $\mathbb{K}[X]^{W_{0}}$. Thus the action of $\mathbb{K}[X]^{W_{0}}$ on $\mathcal{F}_{\ell}=V / I$ is well-defined.

Remark 1.2. One might be tempted to try to define an action of $\mathbb{K}[X]$ on $\mathcal{F}_{\ell}$ by $X^{\mu} \cdot|\gamma\rangle=|\gamma+\ell \mu\rangle$ for $\mu, \gamma \in \mathfrak{a}_{\mathbb{Z}}^{*}$ but this action is not well-defined. For example in the $G=S L_{2}$ case with $\ell=5$ pictured after (1.3), then $|-1\rangle=\left|s_{1} \circ(-1)\right\rangle=-|-1\rangle$ so that $|-1\rangle=0$ and $0=X^{\omega_{1}} \cdot|-1\rangle=|5-1\rangle=|4\rangle$, which is a contradiction to (1.6). On the other hand $0=\left(X^{-\omega_{1}}+X^{\omega_{1}}\right) \cdot|-1\rangle=|-5-1\rangle+|4\rangle=0$, as it should be.

Remark 1.3. The representation theoretic source of the level $(-\ell-h)$-action of (1.12) is the equivalence of categories in Theorem 4.1. If $\mathfrak{h}^{*}=\mathbb{C} \delta+\mathfrak{a}^{*}+\mathbb{C} \Lambda_{0}$ is the Cartan of the affine Kac-Moody Lie algebra $\mathfrak{g}$ associated to $\mathfrak{g}$ (where $\delta$ is the imaginary root and $\Lambda_{0}$ is the fundamental weight corresponding to the additional node of the Dynkin diagram), then the affine Weyl group $W=\left\{w t_{\mu} \mid w \in W_{0}, t_{\mu}\right\}$ acts on $\mathfrak{h}^{*}$ as follows (see $[\mathrm{Kac},(6.5 .2)]$ ): the action of a translation $t_{\mu}$ is given by

$$
\begin{aligned}
& t_{\mu}\left(a \delta+\lambda+m \Lambda_{0}\right)=\left(a-\langle\lambda, \mu\rangle-\frac{1}{2} m\langle\mu, \mu\rangle\right) \delta+\lambda+m \mu+m \Lambda_{0}, \quad \text { and } \\
& w\left(a \delta+\lambda+m \Lambda_{0}\right)=a \delta+w \lambda+m \Lambda_{0} \text { for } w \in W_{0}, \text { the finite Weyl group. }
\end{aligned}
$$

The level $-\ell-h$ subset of $\mathfrak{h}^{*}$ is $\left\{a \delta+\lambda+(-\ell-h) \Lambda_{0} \mid a \in \mathbb{C}, \lambda \in \mathfrak{a}^{*}\right\}$ and the dot action is given by $u \circ \mu=u(\mu+\hat{\rho})-\hat{\rho}$, where $\hat{\rho} \in \mathfrak{h}^{*}$ is such that $\left\langle\hat{\rho}, \alpha_{i}^{\vee}\right\rangle=$ 1 for $i \in\{0, \ldots, n\}$. In Theorem 4.1 the affine Lie algebra representations are representations of $\mathfrak{g}^{\prime}$ (the Lie algebra $\mathfrak{g}$ without the derivation) and so the desired action of the affine Weyl group is $\bmod \delta$. A short computation (see [LRS, $(3,19)]$ ) using that $\hat{\rho}=\rho+h \Lambda_{0}$ gives

$$
\left(t_{\mu} w\right) \circ\left(\lambda+(-\ell-h) \Lambda_{0}\right)=(w \circ \lambda)-\ell \mu+(-\ell-h) \Lambda_{0} \bmod \delta .
$$


Ignoring the $(-\ell-h) \Lambda_{0}$ this is, except for the appearance $w_{0}$, the formula for the level $(-\ell-h)$ dot action on $\mathfrak{a}^{*}$ which is used in (1.12). Working with representations of $\mathfrak{g}$ that are negative level, the fundamental alcove sits in the antidominant chamber instead of the dominant chamber (see (2.10) and (2.14) below), and this is the source of the $w_{0}$ in (1.12).

1.2. The product theorem. Let $\mathfrak{g}$ be the Lie algebra of the reductive group $G$ alluded to in (1.1). For $\lambda \in\left(\mathfrak{a}_{\mathbb{Z}}^{*}\right)^{+}$let $L_{\mathfrak{g}}(\lambda)$ be the irreducible $U(\mathfrak{g})$-module of highest weight $\lambda$ and let $B(\lambda)$ be the crystal of Lakshmibai-Seshadri paths (LS paths) for the representation $L_{\mathfrak{g}}(\lambda)$,

$B(\lambda)=\{$ LS paths $p$ of type $\lambda\}$ and $\operatorname{wt}(p)$ denotes the endpoint of $p ;$

see $[\mathrm{Ra}, \S 5]$. The Weyl character corresponding to $\lambda$ is the element of $\mathbb{K}[X]^{W_{0}}$ given by

$$
s_{\lambda}=\operatorname{char}\left(L_{\mathfrak{g}}(\lambda)\right)=\frac{\sum_{w \in W_{0}} \operatorname{det}(w) X^{w \circ \lambda}}{\sum_{w \in W_{0}} \operatorname{det}(w) X^{w \circ 0}}=\sum_{p \in B(\lambda)} X^{\mathrm{wt}(p)} .
$$

An $\ell$-restricted dominant integral weight is $\lambda_{0} \in\left(\mathfrak{a}_{\mathbb{Z}}^{*}\right)^{+}$such that $\left\langle\lambda_{0}, \alpha_{i}^{\vee}\right\rangle\langle\ell$ for $i \in\{1, \ldots, n\}$. In other words, if $\omega_{1}, \ldots, \omega_{n}$ are the fundamental weights for $\mathfrak{g}$, then a weight $\lambda_{0} \in\left(\mathfrak{a}_{\mathbb{Z}}^{*}\right)^{+}$is $\ell$-restricted if $\lambda_{0}$ is an element of

$$
\Pi_{\ell}=\left\{a_{1} \omega_{1}+\cdots+a_{n} \omega_{n} \mid a_{1}, \ldots, a_{n} \in\{0,1, \ldots, \ell-1\}\right\} .
$$

Theorem 1.4. Let $\lambda \in\left(\mathfrak{a}_{\mathbb{Z}}^{*}\right)^{+}$be a dominant integral weight and write

$$
\lambda=\ell \lambda_{1}+\lambda_{0} \quad \text { with } \lambda_{0} \in \Pi_{\ell} \text { and } \lambda_{1} \in\left(\mathfrak{a}_{\mathbb{Z}}^{*}\right)^{+} .
$$

Then, with $C_{\lambda} \in \mathcal{F}_{\ell}$ as in (1.7) and the $\mathbb{K}[X]^{W_{0}}$-action on $\mathcal{F}_{\ell}$ as in Proposition 1.1 ,

$$
C_{\lambda}=s_{\lambda_{1}^{*}} \cdot C_{\lambda_{0}} .
$$

Proof. The proof is accomplished in two steps:

(a) Show that $s_{\lambda_{1}^{*}} \cdot C_{\lambda_{0}}$ is bar invariant.

(b) Show that $s_{\lambda_{1}^{*}} \cdot C_{\lambda_{0}}=|\lambda\rangle+\sum_{\mu \neq \lambda} c_{\mu}|\mu\rangle$ with $c_{\mu} \in t^{\frac{1}{2}} \mathbb{Z}\left[t^{\frac{1}{2}}\right]$.

Proof of (a). The bar involution and $N_{\gamma}$ are defined in (1.4). Since $\left\langle-\ell w_{0} \mu, \alpha^{\vee}\right\rangle \in$ $\ell \mathbb{Z}$, then

$$
\begin{aligned}
N_{\gamma-\ell w_{0} \mu}=\operatorname{Card}\left\{\alpha \in R^{+} \mid\left\langle\gamma-\ell w_{0} \mu+\rho, \alpha^{\vee}\right\rangle \in \ell \mathbb{Z}\right\} & \\
& =\operatorname{Card}\left\{\alpha \in R^{+} \mid\left\langle\gamma+\rho, \alpha^{\vee}\right\rangle \in \ell \mathbb{Z}\right\}=N_{\gamma} .
\end{aligned}
$$

Thus

$$
\begin{aligned}
\overline{X^{\mu} \cdot|\gamma\rangle} & =\overline{\left|\gamma-\ell w_{0} \mu\right\rangle}=(-1)^{\ell\left(w_{0}\right)}\left(t^{-\frac{1}{2}}\right)^{\ell\left(w_{0}\right)-N_{\gamma-\ell w_{0} \mu}}\left|w_{0} \circ\left(\gamma-\ell w_{0} \mu\right)\right\rangle \\
& =(-1)^{\ell\left(w_{0}\right)}\left(t^{-\frac{1}{2}}\right)^{\ell\left(w_{0}\right)-N_{\gamma-\ell w_{0} \mu}}\left|w_{0}(\gamma+\rho)-\rho-\ell \mu\right\rangle \\
& =(-1)^{\ell\left(w_{0}\right)}\left(t^{-\frac{1}{2}}\right)^{\ell\left(w_{0}\right)-N_{\gamma-\ell w_{0} \mu}}\left|w_{0} \circ \gamma-\ell \mu\right\rangle \\
& =(-1)^{\ell\left(w_{0}\right)}\left(t^{-\frac{1}{2}}\right)^{\ell\left(w_{0}\right)-N_{\gamma}}\left|w_{0} \circ \gamma-\ell \mu\right\rangle \\
& =(-1)^{\ell\left(w_{0}\right)}\left(t^{-\frac{1}{2}}\right)^{\ell\left(w_{0}\right)-N_{\gamma}} X^{w_{0} \mu} \cdot\left|w_{0} \circ \gamma\right\rangle=X^{w_{0} \mu} \cdot \overline{|\gamma\rangle}
\end{aligned}
$$


and since $s_{\lambda_{1}^{*}}$ is $W_{0}$-invariant,

$$
\overline{s_{\lambda_{1}^{*}} \cdot C_{\lambda_{0}}}=\left(w_{0} s_{\lambda_{1}^{*}}\right) \cdot \overline{C_{\lambda_{0}}}=s_{\lambda_{1}^{*}} \cdot C_{\lambda_{0}} .
$$

Proof of (b). Let $\lambda=\lambda_{0}+\ell \lambda_{1}$ as in the statement of the theorem and let $a \equiv b$ mean $a=b \bmod t^{\frac{1}{2}}$ (or, more precisely, that $a-b \in \bigoplus_{\lambda \in \mathfrak{a}_{\mathbb{Z}}^{*}} t^{\frac{1}{2}} \mathbb{Z}\left[t^{\frac{1}{2}}\right]|\lambda\rangle$ ). The proof will rely on the following lemma.

Lemma 1.5. Keeping the notation of Theorem 1.4,

$$
\left|\lambda_{0}+\ell \nu\right\rangle \equiv-\left|\lambda_{0}+\ell\left(s_{i} \circ \nu\right)\right\rangle \quad \text { for } \nu \in \mathfrak{a}_{\mathbb{Z}} .
$$

Proof. By the second formula in (1.7),

$$
s_{\lambda_{1}^{*}} \cdot C_{\lambda_{0}} \equiv s_{\lambda_{1}^{*}} \cdot\left|\lambda_{0}\right\rangle=\sum_{p \in B\left(\lambda_{1}^{*}\right)} X^{\mathrm{wt}(p)}\left|\lambda_{0}\right\rangle=\sum_{p \in B\left(\lambda_{1}^{*}\right)}\left|\ell \mathrm{wt}(p)^{*}+\lambda_{0}\right\rangle .
$$

By (1.3), if $\lambda \in \mathfrak{a}_{\mathbb{Z}}^{*}$ and $\left\langle\lambda+\rho, \alpha_{i}^{\vee}\right\rangle \geq 0$, then

$$
\left|s_{i} \circ \lambda\right\rangle \equiv \begin{cases}-|\lambda\rangle & \text { if }\left\langle\lambda+\rho, \alpha_{i}^{\vee}\right\rangle \in \ell \mathbb{Z}_{\geq 0}, \\ 0 & \text { if } 0<\left\langle\lambda+\rho, \alpha_{i}^{\vee}\right\rangle<\ell, \\ -\left|\lambda^{(1)}\right\rangle & \text { otherwise, }\end{cases}
$$

where $\lambda^{(1)}=\lambda-j \alpha_{i}$ if $\left\langle\lambda+\rho, \alpha_{i}^{\vee}\right\rangle=k \ell+j$ with $j \in\{0,1, \ldots, \ell-1\}$. Since $\lambda^{(1)}=\lambda$ if $\left\langle\lambda+\rho, \alpha_{i}^{\vee}\right\rangle \in \ell \mathbb{Z}_{\geq 0}$, the first case can be viewed as a special case of the last case to read

$$
\left|s_{i} \circ \lambda\right\rangle \equiv \begin{cases}0 & \text { if } 0<\left\langle\lambda+\rho, \alpha_{i}^{\vee}\right\rangle<\ell, \\ -\left|\lambda^{(1)}\right\rangle & \text { otherwise. }\end{cases}
$$

Assume $\left\langle\nu+\rho, \alpha_{i}^{\vee}\right\rangle \in \mathbb{Z}_{\leq 0}$ and let $\lambda=s_{i} \circ\left(\lambda_{0}+\ell \nu\right)$. Since $\left\langle\rho, \alpha_{i}^{\vee}\right\rangle=1$, then

$$
\begin{aligned}
\left\langle\lambda+\rho, \alpha_{i}^{\vee}\right\rangle & =\left\langle s_{i} \circ\left(\lambda_{0}+\ell \nu\right)+\rho, \alpha_{i}^{\vee}\right\rangle=\left\langle s_{i}\left(\lambda_{0}+\ell \nu+\rho\right), \alpha_{i}^{\vee}\right\rangle=\left\langle\lambda_{0}+\ell \nu+\rho, s_{i} \alpha_{i}^{\vee}\right\rangle \\
& =-\left\langle\lambda_{0}+\ell \nu+\rho, \alpha_{i}^{\vee}\right\rangle=\ell\left(-\left\langle\nu+\rho, \alpha_{i}^{\vee}\right\rangle\right)+\left(\ell-1-\left\langle\lambda_{0}, \alpha_{i}^{\vee}\right\rangle\right) .
\end{aligned}
$$

Since $\lambda_{0} \in \Pi_{\ell}$, then $0 \leq \ell-1-\left\langle\lambda_{0}, \alpha_{i}^{\vee}\right\rangle<\ell$ and so

$$
\begin{aligned}
\lambda^{(1)} & =\lambda-\left(\ell-1-\left\langle\lambda_{0}, \alpha_{i}^{\vee}\right\rangle\right) \alpha_{i}=s_{i} \circ\left(\lambda_{0}+\ell \nu\right)-\left(\ell-1-\left\langle\lambda_{0}, \alpha_{i}^{\vee}\right\rangle\right) \alpha_{i} \\
& =s_{i} \lambda_{0}+\ell s_{i} \nu+s_{i} \rho-\rho-\left(\ell-1-\left\langle\lambda_{0}, \alpha_{i}^{\vee}\right\rangle\right) \alpha_{i} \\
& =\left(\lambda_{0}-\left\langle\lambda_{0}, \alpha_{i}^{\vee}\right\rangle \alpha_{i}\right)+\ell\left(s_{i} \nu+s_{i} \rho-\rho\right)+(\ell-1) \alpha_{i}-\left(\ell-1-\left\langle\lambda_{0}, \alpha_{i}^{\vee}\right\rangle\right) \alpha_{i} \\
& =\lambda_{0}+\ell\left(s_{i} \circ \nu\right) .
\end{aligned}
$$

Thus, since $s_{i} \circ \lambda=\lambda_{0}+\ell \nu$,

$$
\left|\lambda_{0}+\ell \nu\right\rangle \equiv \begin{cases}0 & \text { if }\left\langle\nu+\rho, \alpha_{i}^{\vee}\right\rangle=0, \\ -\left|\lambda_{0}+\ell\left(s_{i} \circ \nu\right)\right\rangle & \text { if }\left\langle\nu+\rho, \alpha_{i}^{\vee}\right\rangle<0 .\end{cases}
$$

Since $s_{i} \circ \nu=\nu$ when $\left\langle\nu+\rho, \alpha_{i}^{\vee}\right\rangle=0$, then $\left|\lambda_{0}+\ell \nu\right\rangle \equiv-\left|\lambda_{0}+\ell\left(s_{i} \circ \nu\right)\right\rangle$ when $\left\langle\nu+\rho, \alpha_{i}^{\vee}\right\rangle \in \mathbb{Z}_{\leq 0}$ and, replacing $\nu$ by $s_{i} \circ \nu$, gives $\left|\lambda_{0}+\ell \nu\right\rangle \equiv-\left|\lambda_{0}+\ell\left(s_{i} \circ \nu\right)\right\rangle$ for $\left\langle\nu+\rho, \alpha_{i}^{\vee}\right\rangle \in \mathbb{Z}_{\geq 0}$. Thus

$$
\left|\lambda_{0}+\ell \nu\right\rangle \equiv-\left|\lambda_{0}+\ell\left(s_{i} \circ \nu\right)\right\rangle \quad \text { for } \nu \in \mathfrak{a}_{\mathbb{Z}},
$$

completing the proof of Lemma 1.5 . 
Let us now complete the proof of (b) of Theorem 1.4. Follow [Ra, proof of Theorem 5.5] (see also [Li, proof of Theorem 9.1]) to define an involution $\iota$ on the set $B\left(\lambda_{1}^{*}\right) \backslash\left\{p_{\lambda_{1}^{*}}^{+}\right\}$, where $p_{\lambda_{1}^{*}}^{+}$is the unique highest weight path in $B\left(\lambda_{1}^{*}\right)$.

Let $p \in B\left(\lambda_{1}^{*}\right)$ and $p \neq p_{\lambda_{1}^{*}}^{+}$. Since $p \neq p_{\lambda_{1}^{*}}^{+}$the path $p$ crosses a wall out of the fundamental chamber at some point during its trajectory. Let $r$ be such that the first time $p$ leaves the dominant chamber is by crossing the hyperplane $\left\{x \in \mathfrak{a}_{\mathbb{R}}^{*} \mid\left\langle x, \alpha_{r}^{\vee}\right\rangle=0\right\}$. Letting $\tilde{e}_{r}$ and $\tilde{f}_{r}$ denote the root operators on $B\left(\lambda_{1}^{*}\right)$, the $r$-string containing $p$ is

$$
S_{r}(p)=\left\{q \in B\left(\lambda_{1}^{*}\right) \mid q=\tilde{e}_{r}^{k} p \text { or } q=\tilde{f}_{r}^{k} p \text { where } k \in \mathbb{Z}_{\geq 0}\right\} .
$$

Let

$$
\iota(p) \text { be the element of } S_{r}(p) \text { such that } \mathrm{wt}(\iota(p))=s_{r} \circ \mathrm{wt}(p) .
$$

Note that since $p$ leaves the fundamental chamber at the wall corresponding to $\alpha_{r}^{\vee}$ then $\tilde{e}_{r} p \neq 0$ (i.e., $p$ is not the head of its $r$-string) and so $s_{r} \circ p$ is well-defined. It should be noted that this dot action on paths is different from the classical Weyl group action on the crystal. By Lemma 1.5.

$\left|\lambda_{0}+\ell \mathrm{wt}(p)^{*}\right\rangle \equiv-\left|\lambda_{0}+\ell\left(s_{r}^{*} \circ \mathrm{wt}(p)^{*}\right)\right\rangle=-\left|\lambda_{0}+\ell\left(s_{r} \circ \mathrm{wt}(p)\right)^{*}\right\rangle=-\left|\lambda_{0}+\ell \mathrm{wt}(\iota(p))^{*}\right\rangle$, and so the map $\iota$ partitions the set $B\left(\lambda_{1}^{*}\right) \backslash\left\{p_{\lambda_{1}^{*}}^{+}\right\}$into pairs $\{p, \iota(p)\}$ which cancel each other in the mod $t^{\frac{1}{2}}$ straightening of the terms of $s_{\lambda_{1}^{*}} \cdot C_{\lambda_{0}}$ in (1.16). Thus

$$
s_{\lambda_{1}^{*}} \cdot C_{\lambda_{0}} \equiv\left|\ell\left(\lambda_{1}^{*}\right)^{*}+\lambda_{0}\right\rangle=\left|\lambda_{0}+\ell \lambda_{1}\right\rangle \quad \text { which proves (b). }
$$

\section{The Casselman-Shalika formula}

In order to establish the Casselman-Shalika formula it is necessary to use the connection between the abstract Fock space $\mathcal{F}_{\ell}$ and the affine Hecke algebra $H$. Let us recall this relationship from [LRS].

2.1. The affine Hecke algebra $H$. Keep the notation for the finite Weyl group $W_{0}$, the simple reflections $s_{1}, \ldots, s_{n}$, and the weight lattice $\mathfrak{a}_{\mathbb{Z}}^{*}$ as in (1.1). For $i, j \in\{1, \ldots, n\}$ with $i \neq j$, let $m_{i j}$ denote the order of $s_{i} s_{j}$ in $W_{0}$ so that $s_{i}^{2}=1$ and $\left(s_{i} s_{j}\right)^{m_{i j}}=1$ are the relations for the Coxeter presentation of $W_{0}$. Let $\mathbb{K}=$ $\mathbb{Z}\left[t^{\frac{1}{2}}, t^{-\frac{1}{2}}\right]$. The affine Hecke algebra is

$$
H=\mathbb{K}-\operatorname{span}\left\{X^{\mu} T_{w} \mid \mu \in \mathfrak{a}_{\mathbb{Z}}^{*}, w \in W_{0}\right\},
$$

with $\mathbb{K}$-basis $\left\{X^{\mu} T_{w} \mid \mu \in \mathfrak{a}_{\mathbb{Z}}^{*}, w \in W_{0}\right\}$ and relations

$$
\left(T_{s_{i}}-t^{\frac{1}{2}}\right)\left(T_{s_{i}}+t^{-\frac{1}{2}}\right)=0, \quad \underbrace{T_{s_{i}} T_{s_{j}} T_{s_{i}} \ldots}_{m_{i j} \text { factors }}=\underbrace{T_{s_{j}} T_{s_{i}} T_{s_{j}} \ldots}_{m_{i j} \text { factors }},
$$

$$
X^{\lambda+\mu}=X^{\lambda} X^{\mu}, \quad \text { and } \quad T_{s_{i}} X^{\lambda}-X^{s_{i} \lambda} T_{s_{i}}=\left(t^{\frac{1}{2}}-t^{-\frac{1}{2}}\right)\left(\frac{X^{\lambda}-X^{s_{i} \lambda}}{1-X^{-\alpha_{i}}}\right)
$$

for $i, j \in\{1, \ldots, n\}$ with $i \neq j$ and $\lambda, \mu \in \mathfrak{a}_{\mathbb{Z}}^{*}$. The bar involution on $H$ is the $\mathbb{Z}$-linear automorphism ${ }^{-}: H \rightarrow H$ given by

$$
\overline{t^{\frac{1}{2}}}=t^{-\frac{1}{2}}, \quad \overline{T_{s_{i}}}=T_{s_{i}}^{-1}, \quad \text { and } \quad \overline{X^{\lambda}}=T_{w_{0}} X^{w_{0} \lambda} T_{w_{0}}^{-1}
$$


for $i=1, \ldots, n$ and $\lambda, \mu \in \mathfrak{a}_{\mathbb{Z}}^{*}$. For $\mu \in \mathfrak{a}_{\mathbb{Z}}^{*}$ and $w \in W_{0}$ define

$$
X^{t_{\mu} w}=X^{\mu}\left(T_{w^{-1}}\right)^{-1} \quad \text { and } \quad T_{t_{\mu} w}=T_{x} X^{\mu^{+}} T_{w_{\mu^{+}}}\left(T_{w^{-1} x w_{\mu^{+}}}\right)^{-1},
$$

where $\mu^{+}$is the dominant representative of $W_{0} \mu, x \in W_{0}$ is the element of minimal length such that $\mu=x \mu^{+}$, and $w_{\mu^{+}}$is the longest element of the stabilizer $W_{\mu^{+}}=$ $\operatorname{Stab}_{W_{0}}\left(\mu^{+}\right)$. Define

$$
\varepsilon_{0}=\left(-t^{\frac{1}{2}}\right)^{\ell\left(w_{0}\right)} \sum_{z \in W_{0}}\left(-t^{-\frac{1}{2}}\right)^{\ell(z)} T_{z} \quad \text { and } \quad \mathbf{1}_{0}=\left(t^{-\frac{1}{2}}\right)^{\ell\left(w_{0}\right)} \sum_{z \in W_{0}}\left(t^{\frac{1}{2}}\right)^{\ell(z)} T_{z},
$$

so that

$$
\overline{\varepsilon_{0}}=\varepsilon_{0}, \quad \overline{\mathbf{1}_{0}}=\mathbf{1}_{0}, \quad \text { and } \quad \varepsilon_{0} T_{s_{i}}=-t^{-\frac{1}{2}} \varepsilon_{0}, \quad \text { and } \quad T_{s_{i}} \mathbf{1}_{0}=t^{\frac{1}{2}} \mathbf{1}_{0}
$$

for $i \in\{1, \ldots, n\}$. The algebra $\mathbb{K}[X]$ defined in (1.8) is a subalgebra of $H$ and, by a theorem of Bernstein (see [NR, Theorem 1.4]), the center of $H$ is the ring of symmetric functions,

$$
Z(H)=\mathbb{K}[X]^{W_{0}} .
$$

Remark 2.1. Formulas (2.4) and (2.5) are just a reformulation of the usual bar involution and the conversion between the Bernstein and Coxeter presentations of the affine Hecke algebra (see for example [NR, Lemma 2.8 and (1.22)]).

2.2. The relation between $H$ and the abstract Fock space $\mathcal{F}_{\ell}$. In this subsection we follow [LRS, §4.2]. The affine Weyl group is

(2.8) $W=\left\{t_{\mu} w \mid \mu \in \mathfrak{a}_{\mathbb{Z}}^{*}, w \in W_{0}\right\} \quad$ with $\quad t_{\mu} t_{\nu}=t_{\mu+\nu} \quad$ and $\quad w t_{\mu}=t_{w \mu} w$

for $\mu, \nu \in \mathfrak{a}_{\mathbb{Z}}^{*}$ and $w \in W_{0}$. Let $\varphi^{\vee}$ and $h$ be as in (1.11). For $\ell \in \mathbb{Z}_{>0}$, the level $(-\ell-h)$ dot action of $W$ on $\mathfrak{a}_{\mathbb{Z}}^{*}$ is given by

$$
\left(t_{\mu} w\right) \circ \lambda=(w \circ \lambda)-\ell \mu=w(\lambda+\rho)-\rho-\ell \mu
$$

for $\mu \in \mathfrak{a}_{\mathbb{Z}}^{*}, w \in W_{0}$, and $\lambda \in \mathfrak{a}_{\mathbb{Z}}^{*}$. Note that this is an extension of the dot action of $W_{0}$ given in (1.2). Define

$$
A_{-\ell-h}=\left\{\nu \in \mathfrak{a}_{\mathbb{Z}}^{*} \mid\left\langle\nu, \varphi^{\vee}\right\rangle \geq-\ell-1 \text { and }\left\langle\nu, \alpha_{i}^{\vee}\right\rangle \leq-1 \text { for } i \in\{1, \ldots, n\}\right\}
$$

and

$$
\mathcal{P}_{-\ell-h}^{+}=\bigoplus_{\nu \in A_{-\ell-h}} \varepsilon_{0} H \mathbf{p}_{\nu}
$$

where $\varepsilon_{0}$ is as in (2.6) and $\mathbf{p}_{\nu}$ are formal symbols indexed by $\nu \in A_{-\ell-h}$ satisfying

$$
\overline{\mathbf{p}_{\nu}}=\mathbf{p}_{\nu} \quad \text { and } \quad T_{y} \mathbf{p}_{\nu}=\left(t^{\frac{1}{2}}\right)^{\ell(y)} \mathbf{p}_{\nu} \text { for } y \in W_{\nu},
$$

where $W_{\nu}=\operatorname{Stab}_{W}(\nu)$ is the stabilizer of $\nu$ under the level $(-\ell-h)$ dot action of $W$ on $\mathfrak{a}_{\mathbb{Z}}^{*}$. (Warning: There is a slight conflict of notation here with the notation $W_{0}$ for the finite Weyl but this should not cause confusion.) Define a bar involution $(2.12)$

$$
: \mathcal{P}_{-\ell-h}^{+} \rightarrow \mathcal{P}_{-\ell-h}^{+} \quad \text { by } \quad \overline{\varepsilon_{0} f \mathbf{p}_{\nu}}=\varepsilon_{0} \bar{f} \mathbf{p}_{\nu} \quad \text { for } \nu \in A_{-\ell-h} \text { and } f \in H .
$$

For $\lambda \in \mathfrak{a}_{\mathbb{Z}}^{*}$ define

$$
\left[X_{\lambda}\right]=\left[X_{w_{0} v \circ \nu}\right]=\varepsilon_{0} X^{v} \mathbf{p}_{\nu}, \quad \text { where } \quad \lambda=w_{0} v \circ \nu \quad \text { with } \nu \in A_{-\ell-h},
$$


and $v \in W$ is such that $X^{v u}=X^{v} T_{u}$ for any $u \in W_{\nu}$. It is helpful to stress that the $(-\ell-h)$ dot action of (2.9) applies here so that, when $v=t_{\mu} w$ with $\mu \in \mathfrak{a}_{\mathbb{Z}}^{*}$ and $w \in W_{0}$, then $\lambda=-\ell w_{0} \mu+\left(w_{0} w\right) \circ \nu$ and

$$
\left[X_{\lambda}\right]=\left[X_{-\ell w_{0} \mu+\left(w_{0} w\right) \circ \nu}\right]=\varepsilon_{0} X^{t_{\mu} w} \mathbf{p}_{\nu}=\varepsilon_{0} X^{\mu}\left(T_{w^{-1}}\right)^{-1} \mathbf{p}_{\nu}
$$

With this notation, a main result of [LRS] is the following.

Theorem 2.2 (see [LRS, Theorem 4.7]). Let $\leq$ be the dominance order on the set $\left(\mathfrak{a}_{\mathbb{Z}}^{*}\right)^{+}$of dominant integral weights. Then the $\mathbb{K}$-linear map $\Phi: \mathcal{F}_{\ell} \rightarrow \mathcal{P}_{-\ell-h}^{+}$given by

$$
\Phi(|\lambda\rangle)=\left[X_{\lambda}\right] \quad \text { for } \lambda \in \mathfrak{a}_{\mathbb{Z}}^{*},
$$

is a well-defined $\mathbb{K}$-module isomorphism satisfying $\overline{\Phi(f)}=\Phi(\bar{f})$.

Since elements of $Z(H)=\mathbb{K}[X]^{W_{0}}$ commute with $\varepsilon_{0}$ there is a $\mathbb{K}[X]^{W_{0}}$-action on $\mathcal{P}_{-\ell-h}$ by left multiplication. The pullback of this action by the isomorphism $\Phi$ is the source of the $\mathbb{K}[X]^{W_{0}}$ action on $\mathcal{F}_{\ell}$ given in Proposition 1.1.

$$
z \Phi(f)=\Phi(z f) \quad \text { for } z \in Z(H)=\mathbb{K}[X]^{W_{0}} \text { and } f \in \mathcal{F}_{\ell} .
$$

2.3. Deducing the Casselman-Shalika formula. For $\mu \in \mathfrak{a}_{\mathbb{Z}}^{*}$ define the "Whittaker function" $A_{\mu} \in \varepsilon_{0} H \mathbf{1}_{0}$ by

$$
A_{\mu}=\varepsilon_{0} X^{\mu} \mathbf{1}_{0} .
$$

See, for example, [HKP, $\S 6]$ for the connection between $p$-adic groups and the affine Hecke algebra and the explanation of why $A_{\mu}$ is equivalent to the data of a (spherical) Whittaker function for a $p$-adic group. As proved carefully in $[\mathrm{NR}$, Theorem 2.7], it follows from (2.6) and (2.3) that

$$
\varepsilon_{0} H \mathbf{1}_{0} \text { has } \mathbb{K} \text {-basis } \quad\left\{A_{\lambda+\rho} \mid\left\langle\lambda+\rho, \alpha_{i}\right\rangle \in \mathbb{Z}_{\geq 0} \text { for } i \in\{1, \ldots, n\}\right\} .
$$

Following [NR, Theorem 2.4], the Satake isomorphism, $\mathbb{K}[X]^{W_{0}} \cong \mathbf{1}_{0} H \mathbf{1}_{0}$, and the Casselman-Shalika formula, $A_{\lambda+\rho}=s_{\lambda} A_{\rho}$, can be formulated by the following diagram of vector space (free $\mathbb{K}$-module) isomorphisms:

$$
\begin{array}{clcll}
Z(H)=\mathbb{K}[X]^{W_{0}} & \stackrel{\sim}{\longrightarrow} \mathbf{1}_{0} H \mathbf{1}_{0} & \stackrel{\sim}{\longrightarrow} \varepsilon_{0} H \mathbf{1}_{0} \\
f & \longmapsto & f \mathbf{1}_{0} & \longmapsto & A_{\rho} f \mathbf{1}_{0} \\
s_{\lambda} & \longmapsto & s_{\lambda} \mathbf{1}_{0} & \longmapsto & A_{\lambda+\rho} .
\end{array}
$$

This diagram has particular importance due to the fact that $\mathbb{K}[X]^{W_{0}}$ is an avatar of the Grothendieck group of the category $\operatorname{Rep}(G)$ of finite dimensional representations of $G$, the spherical Hecke algebra $\mathbf{1}_{0} H \mathbf{1}_{0}$ is a form of the Grothendieck group of $K$-equivariant perverse sheaves on the loop Grassmanian $G r$ for the Langlands dual group $G^{\vee}$, and $\varepsilon_{0} H \mathbf{1}_{0}$ is isomorphic to the Grothendieck group of Whittaker sheaves (appropriately formulated $N$-equivariant sheaves on $G r$ ); see [FGV].

Our proof of the Casselman-Shalika formula is accomplished by restricting Theorem 1.4 to the summand in (2.11) corresponding to $-\rho \in A_{-\ell-h}$. We shall identify this summand with $\varepsilon_{0} H \mathbf{1}_{0}$ via the $Z(H)$-isomorphism

$$
\begin{aligned}
& \varepsilon_{0} H \mathbf{1}_{0} \stackrel{\sim}{\longrightarrow} \varepsilon_{0} H \mathbf{p}_{-\rho} \\
& \varepsilon_{0} X^{\mu} \mathbf{1}_{0} \longmapsto \varepsilon_{0} X^{\mu} \mathbf{p}_{-\rho} .
\end{aligned}
$$

Using the level $(-\ell-h)$ dot action of $W$ from (2.9), the stabilizer of $-\rho$ is $W_{0}$ and

$$
W \circ(-\rho)=\left\{t_{-\lambda} \circ(-\rho) \mid \lambda \in \mathfrak{a}_{\mathbb{Z}}^{*}\right\}=\left\{\ell \lambda-\rho \mid \lambda \in \mathfrak{a}_{\mathbb{Z}}^{*}\right\} .
$$


Since $\left\langle(\ell \lambda-\rho)+\rho, \alpha^{\vee}\right\rangle \in \ell \mathbb{Z}$ for $\alpha \in R^{+}$, the straightening law (1.3) for elements of $W \circ(-\rho)$ is

$$
\left|s_{i} \circ(\ell \lambda-\rho)\right\rangle=-|\ell \lambda-\rho\rangle .
$$

Theorem 2.3 (Casselman-Shalika). For $\lambda \in\left(\mathfrak{a}_{\mathbb{Z}}^{*}\right)^{+}$and $\mu \in \mathfrak{a}_{\mathbb{Z}}^{*}$ let $s_{\lambda}$ be the Weyl character as defined in (1.14) and let $A_{\mu}$ be the Whittaker function as defined in (2.17). Then

$$
s_{\lambda} A_{\rho}=A_{\lambda+\rho} .
$$

Proof. Using (2.19),

$\overline{|\ell \lambda-\rho\rangle}=(-1)^{\ell\left(w_{0}\right)}\left(t^{-\frac{1}{2}}\right)^{\ell\left(w_{0}\right)-\ell\left(w_{0}\right)}\left|w_{0} \circ(\ell \lambda-\rho)\right\rangle=(-1)^{\ell\left(w_{0}\right)}\left|w_{0} \circ(\ell \lambda-\rho)\right\rangle=|\ell \lambda-\rho\rangle$ and thus $|\ell \lambda-\rho\rangle$ satisfies the conditions of (1.7) so that

$$
C_{\ell \lambda-\rho}=|\ell \lambda-\rho\rangle, \quad \text { for } \lambda \in\left(\mathfrak{a}_{\mathbb{Z}}^{*}\right)^{+} .
$$

By (2.14) and (2.17),

$$
\left[X_{-\ell w_{0} \mu-\rho}\right]=\varepsilon_{0} X^{\mu} T_{w_{0}}^{-1} \mathbf{p}_{-\rho}=t^{-\ell\left(w_{0}\right) / 2} \varepsilon_{0} X^{\mu} \mathbf{p}_{-\rho}=t^{-\ell\left(w_{0}\right) / 2} A_{\mu} \quad \text { for } \mu \in\left(\mathfrak{a}_{\mathbb{Z}}^{*}\right)^{+} \text {. }
$$

Using (2.16), (2.20), (2.15), and that $w_{0} \rho=-\rho$,

$$
\begin{aligned}
t^{-\ell\left(w_{0}\right) / 2} s_{\lambda} & A_{\rho}=s_{\lambda}\left[X_{-\ell w_{0} \rho-\rho}\right]=s_{\lambda}\left[X_{(\ell-1) \rho}\right]=s_{\lambda} \Phi(|(\ell-1) \rho\rangle)=\Phi\left(s_{\lambda}|(\ell-1) \rho\rangle\right) \\
& =\Phi\left(s_{\lambda} C_{(\ell-1) \rho}\right)=\Phi\left(C_{-\ell w_{0} \lambda+(\ell-1) \rho}\right), \quad \text { by Theorem 1.4 } \\
& =\Phi\left(\left|\left(-\ell w_{0} \lambda\right)+(\ell-1) \rho\right\rangle\right)=\left[X_{-\ell w_{0} \lambda+(\ell-1) \rho}\right]=\left[X_{-\ell w_{0}(\lambda+\rho)-\rho}\right] \\
& =t^{-\ell\left(w_{0}\right) / 2} A_{\lambda+\rho} .
\end{aligned}
$$

\section{QuANTUM GROUPS AND LLT POLYNOMIALS}

In this section we describe the main motivation for Theorem 1.4 namely, the Steinberg-Lusztig tensor product theorem for representations of quantum groups at roots of unity. Then we explain the connection between these results and the theory of LLT polynomials.

3.1. Representations of quantum groups at a root of unity. Let $\stackrel{\mathfrak{g}}{\text { be the }}$ Lie algebra of the group $G$ alluded to in (1.1). Let $q \in \mathbb{C}^{\times}$and let $U_{q}(\mathfrak{g})$ be the Drinfel'd-Jimbo quantum group corresponding to $\mathfrak{\mathfrak { g }}$. Let

$$
\begin{aligned}
& \Delta_{q}(\lambda) \quad \text { the Weyl module for } U_{q}(\mathfrak{g}) \text { of highest weight } \lambda, \\
& L_{q}(\lambda) \quad \text { the simple module for } U_{q}(\mathfrak{g}) \text { of highest weight } \lambda .
\end{aligned}
$$

Let

$K\left(\mathrm{fd}-U_{q}(\mathfrak{g})\right.$-mod) be the free $\mathbb{Z}\left[t^{\frac{1}{2}}, t^{-\frac{1}{2}}\right]$-module generated by symbols $\left[\Delta_{q}(\lambda)\right]$ for $\lambda \in \mathfrak{a}_{\mathbb{Z}}^{*}$. For $\mu \in \mathfrak{a}_{\mathbb{Z}}^{*}$, denote by $W^{\mu}$, resp., ${ }^{\mu} W$, the set of minimal length coset representatives for $W / W_{\mu}$, resp., $W_{\mu} \backslash W$. Define elements $\left[L_{q}\left(w_{0} y \circ \nu\right)\right]$ for $\nu \in A_{-\ell-h}$ and $y \in{ }^{0} W$ such that $w_{0} y \in W^{\nu}$, by the equation

$$
\left[\Delta_{q}\left(w_{0} x \circ \nu\right)\right]=\sum_{y \leq x}\left(\sum_{i \in \mathbb{Z}_{\geq 0}}\left[\frac{\Delta_{q}\left(w_{0} x \circ \nu\right)^{(i)}}{\Delta_{q}\left(w_{0} x \circ \nu\right)^{(i+1)}}: L_{q}\left(w_{0} y \circ \nu\right)\right]\left(t^{\frac{1}{2}}\right)^{i}\right)\left[L_{q}\left(w_{0} y \circ \nu\right)\right],
$$

where $\left[M: L_{q}(\mu)\right]$ denotes the multiplicity of the simple $\mathfrak{g}$-module $L_{q}(\mu)$ of highest weight $\mu$ in a composition series of $M$ and

$$
\Delta_{q}(\lambda)=\Delta_{q}(\lambda)^{(0)} \supseteq \Delta_{q}(\lambda)^{(1)} \supseteq \cdots \quad \text { is the Jantzen filtration of } \Delta_{q}(\lambda)
$$


(see, for example, [Sh, $\S 1.4, \S 2.3$ and $\S 2.10$ and Cor. 2.14] and [JM, $\S 4]$ for the Jantzen filtration in this context).

The combination of [LRS, (3.20)] and [LRS, Theorem 4.7]) is the following connection between the representation theory of the quantum group at a root of unity and the abstract Fock space.

Theorem 3.1. Let $\ell \in \mathbb{Z}_{>0}$ and let $q \in \mathbb{C}^{\times}$such that $q^{2 \ell}=1$. Let $\mathbb{K}=\mathbb{Z}\left[t^{\frac{1}{2}}, t^{-\frac{1}{2}}\right]$. Then the $\mathbb{K}$-linear map given by

$$
\begin{aligned}
& K\left(\mathrm{fd}-U_{q}(\mathfrak{g}) \text {-mod }\right) \quad \stackrel{\Psi_{2}}{\longrightarrow} \quad \mathcal{F}_{\ell} \\
& {\left[\Delta_{q}(\lambda)\right] \quad \longmapsto|\lambda\rangle \text { is a well-defined isomorphism of } \mathbb{Z}\left[t^{\frac{1}{2}}, t^{-\frac{1}{2}}\right] \text {-modules. }} \\
& {\left[L_{q}(\lambda)\right] \quad \longmapsto C_{\lambda}}
\end{aligned}
$$

The enveloping algebra $U(\mathfrak{g})$ has a presentation by generators $e_{1}, \ldots, e_{n}, f_{1}, \ldots$, $f_{n}$, and $h_{1}, \ldots, h_{n}$ and Serre relations and the quantum group $U_{q}(\mathfrak{g})$ has a presentation by generators $E_{1}, \ldots, E_{n}, F_{1}, \ldots, F_{n}$, and $K_{1}, \ldots, K_{n}$ and quantum Serre relations such that, at $q=1, E_{i}$ becomes $e_{i}$ and $F_{i}$ becomes $f_{i}$. Following Lu89. and [CP, Theorem 9.3.12], with appropriate restrictions on $\ell$ as in CP, just before Proposition 9.3.5 and Theorem 9.3.12], the Frobenius map is the Hopf algebra homomorphism

$$
\begin{aligned}
& F r: \quad U_{q}(\mathfrak{g}) \quad \longrightarrow \quad U(\mathfrak{g}) \\
& E_{i}^{(r)} \mapsto \begin{cases}e_{i}^{(r / \ell)} & \text { if } \ell \text { divides } r, \\
0 & \text { otherwise, }\end{cases} \\
& F_{i}^{(r)} \mapsto \begin{cases}f_{i}^{(r / \ell)} & \text { if } \ell \text { divides } r, \\
0 & \text { otherwise, }\end{cases}
\end{aligned}
$$

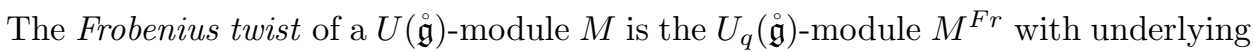
vector space $M$ and $U_{q}(\mathfrak{g})$-action given by

$$
u m=\operatorname{Fr}(u) m \quad \text { for } u \in U_{q}(\mathfrak{g}) \text { and } m \in M .
$$

Theorem 3.2 ([Lu89, Theorem 7.4]; see also [CP, 11.2.9]). Let $\ell \in \mathbb{Z}_{>0}$ and let $\Pi_{\ell}$ be as defined in (1.15). Let $\lambda \in\left(\mathfrak{a}_{\mathbb{Z}}^{*}\right)^{+}$and write

$$
\lambda=\ell \lambda_{1}+\lambda_{0} \quad \text { with } \lambda_{0} \in \Pi_{\ell} \text { and } \lambda_{1} \in\left(\mathfrak{a}_{\mathbb{Z}}^{*}\right)^{+} .
$$

Let $q \in \mathbb{C}^{\times}$be such that $q^{2 \ell}=1$ and let $L_{q}(\lambda)$ denote the simple $U_{q}(\mathfrak{g})$-module of highest weight $\lambda$. Then

$$
L_{q}(\lambda) \cong \Delta\left(\lambda_{1}\right)^{F r} \otimes L_{q}\left(\lambda_{0}\right),
$$

where $\Delta(\mu)$ denotes the irreducible $U(\mathfrak{g})$-module of highest weight $\mu$.

Accepting Theorem 3.1 , Theorem 3.2 is equivalent to the product theorem for abstract Fock space, Theorem 1.4 .

3.2. LLT polynomials for general Lie-type. In [LLT] and [LT, (43)] and [GH, Definition 6.6], the LLT polynomials for type A are defined by

$$
G_{\mu / \nu}^{(\ell)}\left(x, t^{-1}\right) \sum_{T \in S S R T_{\ell}(\mu / \nu)} t^{-\operatorname{spin}(T)} x^{T},
$$

where $S S R T_{\ell}(\mu / \nu)$ is the set of semistandard $\ell$ ribbon tableaux of shape $\mu / \nu$, $\operatorname{spin}(T)$ is the spin of the tableaux $T$ and $X^{T}$ is the weight of the tableaux $T$ 
(see [GH], §6.5] for an efficient review of the combinatorial definitions of semistandard ribbon tableaux, spin, and weight).

In Lecouvey Lcy, there is a definition of LLT polynomials for general Lie-type generalizing the definition of [LLT] from type A which proceeds as follows. Define a $\mathbb{K}$-algebra homomorphism

$$
\begin{aligned}
\psi_{\ell}: \mathbb{K}[X] & \longrightarrow \mathbb{K}[X] \\
X^{\mu} & \longmapsto X^{\ell \mu}
\end{aligned} \text { so that } \quad \psi_{\ell}\left(s_{\lambda}\right)=\operatorname{char}\left(\Delta_{q}(\lambda)^{F r}\right)
$$

in the framework of Theorem 3.2 , Then $[\mathrm{Lcy},(57)]$ defines

$$
G_{\mu}^{\ell}=\sum_{\lambda \in\left(\mathfrak{a}_{\mathbb{Z}}^{*}\right)^{+}} p_{\ell \lambda, \mu} s_{\lambda}
$$

where $p_{\ell \lambda, \mu} \in \mathbb{Z}\left[t^{\frac{1}{2}}\right]$ are as in (1.7). As pointed out in Lcy, Cor. 5.1.3], Theorem 3.1 gives

$$
\begin{aligned}
\psi_{\ell}\left(s_{\lambda}\right) & =\operatorname{char}\left(\Delta(\lambda)^{F r}\right)=\operatorname{char}\left(L_{q}(\ell \lambda)\right) \\
& =\sum_{\mu \in\left(\mathfrak{a}_{\mathbb{Z}}^{*}\right)^{+}} p_{\ell \lambda, \mu}(1) \operatorname{char}\left(\Delta_{q}(\mu)\right)=\sum_{\mu \in\left(\mathfrak{a}_{\mathbb{Z}}^{*}\right)^{+}} p_{\ell \lambda, \mu}(1) s_{\mu} .
\end{aligned}
$$

As explained carefully in [LRS, Theorem 4.8(b)], the polynomials $p_{\ell \lambda, \mu}$ are parabolic singular Kazhdan-Lusztig polynomials.

In GH, Definition 5.12 and Corollary 6.4] there is another definition of LLT polynomials for general Lie-type:

$$
\mathcal{L}_{L, \beta, \gamma}^{G}=t^{l_{\beta-\gamma}+\ell(w)-\ell(v)} \sum_{\lambda \in\left(\mathfrak{a}_{\mathbb{Z}}^{*}\right)^{+}} Q_{\mu \nu}^{\lambda} s_{\lambda}, \quad \text { where } \quad s_{\lambda^{*}} \cdot|\nu\rangle=\sum_{\mu} Q_{\mu \nu}^{\lambda}|\mu\rangle
$$

determine the polynomials $Q_{\nu \mu}^{\lambda}$. Here $G$ is the reductive algebraic group alluded to in (1.1), $L$ is a Levi subgroup of $G$ with Weyl group $W_{\nu}, l_{\beta-\gamma}$ is the nonnegative integer defined in [GH, Remark 5.10], and

$\mu=v \circ(\eta+\ell \beta)$ and $\nu=w \circ(\eta+\ell \gamma)$, where $v \in W_{0} t_{\beta} W_{\eta}$ and $\quad w \in W_{0} t_{\gamma} W_{\eta}$ are minimal representatives. At this point, the reader's discomfort occurring from the transitions between $\beta$ and $\gamma$ and $v$ and $w$ and $\mu$ and $\nu$ is mitigated by recognizing that the relation between these two definitions occurs in the special case $\nu=0$ : Theorem 1.4 and (2.20) and the definition of $Q_{\mu \nu}^{\lambda}$ in (3.4) give

$C_{\ell \lambda}=s_{\lambda^{*}} \cdot C_{0}=s_{\lambda^{*}} \cdot|0\rangle=\sum_{\mu} Q_{\mu 0}^{\lambda}|\mu\rangle$, and comparing with (1.7) gives $p_{\ell \lambda, \mu}=Q_{\mu, 0}^{\lambda}$

and specifies the close relationship between $G_{\mu}^{\ell}$ and $\mathcal{L}_{L, \beta, \gamma}^{G}$ which occurs at $\nu=0$. They are the same up to a power of $t$.

\section{Tensor Product theorem on AFFine Lie ALGEBra Representations}

Let $\mathfrak{g}$ be the Lie algebra of $G$ and let $\mathfrak{g}=\mathfrak{g} \otimes_{\mathbb{C}} \mathbb{C}\left[\epsilon, \epsilon^{-1}\right]+\mathbb{C} K+\mathbb{C} d$ be the corresponding affine Kac-Moody Lie algebra (see [Kac, §6.2] - we follow the notation of [LRS, (3.17)]). Let $\ell \in \mathbb{Z}_{>0}$ and let $h$ be the dual Coxeter number. As explained in [LRS, Theorem 3.2], an important result of Kazhdan-Lusztig establishes a relation between level $(-\ell-h)$-representations in parabolic category $\mathcal{O}_{\mathfrak{g}}^{\mathfrak{g}}$ for the affine Lie algebra and the finite dimensional representations of the quantum group $U_{q}(\mathfrak{g})$ with $q^{2 \ell}=1$. 
Let

$$
\mathfrak{g}^{\prime}=[\mathfrak{g}, \mathfrak{g}]=\mathfrak{g} \otimes_{\mathbb{C}} \mathbb{C}\left[\epsilon, \epsilon^{-1}\right]+\mathbb{C} K .
$$

By restriction, the modules in $\mathcal{O}_{\mathfrak{g}}^{\mathfrak{g}}$ are $\mathfrak{g}^{\prime}$-modules. Let $\Lambda_{0}$ be the fundamental weight of the affine Lie algebra so that $L\left(c \Lambda_{0}+\lambda\right)$ is an irreducible highest weight $\mathfrak{g}$-module of level $c$ (i.e., $K$ acts by the constant $c$ ).

Theorem 4.1 ([KL94, Theorem 38.1]). There is an equivalence of categories

$$
\begin{array}{cccc}
\left\{\begin{array}{c}
\text { finite length } \mathfrak{g}^{\prime} \text {-modules } \\
\text { of level }-\ell-h \text { in } \mathcal{O}_{\mathfrak{g}}^{\mathfrak{g}}
\end{array}\right\} & \stackrel{\Psi_{1}}{\longrightarrow} & \left\{\begin{array}{c}
\text { finite dimensional } U_{q}(\mathfrak{g}) \text {-modules } \\
\text { with } q^{2 \ell}=1
\end{array}\right\} \\
\Delta_{\mathfrak{g}}^{\mathfrak{g}}\left((-\ell-h) \Lambda_{0}+\lambda\right) & \longmapsto & \Delta_{q}(\lambda) \\
L\left((-\ell-h) \Lambda_{0}+\lambda\right) & \longmapsto & L_{q}(\lambda) .
\end{array}
$$

This statement of Theorem 4.1 is for the simply-laced (symmetric) case. With the proper modifications to this statement the result holds for non-simply-laced cases as well; see [Lu94, §8.4] and Lu95.

Let $\ell \lambda-\rho \in\left(\mathfrak{a}_{\mathbb{Z}}^{*}\right)^{+}$. Under the composition of the map $\Psi_{1}$ in Theorem 4.1 and the map $\Psi_{2}$ from Theorem 3.1 .

$$
\Psi_{2}\left(\Psi_{1}\left(\left[L\left((-\ell-h) \Lambda_{0}+\ell \lambda\right)-\rho\right]\right)\right)=\Psi_{2}\left(\left[L_{q}(\ell \lambda-\rho)\right]\right)=C_{\ell \lambda-\rho}=|\ell \lambda-\rho\rangle .
$$

Thus it follows from Theorem 4.1, Theorem 3.1 and (2.20) that

$$
\begin{aligned}
L\left((-\ell-h) \Lambda_{0}+\ell \lambda-\rho\right) & =\Delta_{\mathfrak{g}}^{\mathfrak{g}}\left((-\ell-h) \Lambda_{0}+\ell \lambda-\rho\right) \\
& =\operatorname{Ind}_{\mathfrak{g}_{0}^{\circ}+\mathfrak{b}}^{\mathfrak{g}}\left(L_{\mathfrak{g}}(\ell \lambda-\rho)\right) \cong U(\mathfrak{g}) \otimes_{U(\mathfrak{k})} L_{\mathfrak{g}}(\ell \lambda-\rho),
\end{aligned}
$$

where

$$
\mathfrak{k}=\bigoplus_{k \in \mathbb{Z} \geq 0} \epsilon^{k}\left(\mathfrak{a} \oplus \bigoplus_{\alpha \in R^{+}} \stackrel{\circ}{\alpha}_{\alpha}+\stackrel{\mathfrak{g}}{-\alpha}_{-\alpha}\right) \quad \text { with } R^{+} \text {the set of positive roots of } \mathfrak{\mathfrak { g }} .
$$

As given in (1.14), the Weyl character formula for the $\mathfrak{\mathfrak { g }}$-module $L_{\mathfrak{g}}(\ell \lambda-\rho)$ is

$$
\begin{aligned}
\operatorname{char}\left(L_{\mathfrak{g}}(\ell \lambda-\rho)\right)=s_{\ell \lambda-\rho} & =\left(\prod_{\alpha \in R^{+}} \frac{1}{1-X^{-\alpha}}\right) \cdot \sum_{w \in W_{0}} \operatorname{det}(w) X^{w \circ(\ell \lambda-\rho)} \\
& =\left(\prod_{\alpha \in R^{+}} \frac{1}{1-X^{-\alpha}}\right) \cdot \sum_{w \in W_{0}} \operatorname{det}(w) X^{w(\ell \lambda)-\rho} .
\end{aligned}
$$

Letting $q=e^{\delta}, n=\operatorname{dim}(\mathfrak{a})$, and using the Poincaré-Birkhoff-Witt theorem, the character of the $\mathfrak{g}$-module in (4.1) is

$$
\begin{aligned}
\operatorname{char}\left(L\left((-\ell-h) \Lambda_{0}+\ell \lambda-\rho\right)\right) & =\operatorname{char}\left(\Delta_{\mathfrak{g}}^{\mathfrak{g}}\left((-\ell-h) \Lambda_{0}+\ell \lambda-\rho\right)\right) \\
& =\operatorname{char}\left(U(\mathfrak{g}) \otimes_{U(\mathfrak{k})} L_{\mathfrak{g}}(\ell \lambda-\rho)\right) \\
= & s_{\ell \lambda-\rho} \prod_{k \in \mathbb{Z}_{>0}}\left(\frac{1}{\left(1-q^{-k}\right)^{n}} \prod_{\alpha \in R^{+}} \frac{1}{1-q^{-k} X^{\alpha}} \cdot \frac{1}{1-q^{-k} X^{-\alpha}}\right) \\
= & \left(\prod_{k \in \mathbb{Z}_{>0}} \frac{1}{\left(1-q^{-k}\right)^{n}}\right)\left(\prod_{k \in \mathbb{Z}_{>0}} \prod_{\alpha \in R^{+}} \frac{1}{1-q^{-k} X^{\alpha}}\right) \\
& \cdot\left(\prod_{k \in \mathbb{Z}_{\geq 0}} \prod_{\alpha \in R^{+}} \frac{1}{1-q^{-k} X^{-\alpha}}\right)\left(\sum_{w \in W_{0}} \operatorname{det}(w) X^{w(\ell \lambda)-\rho}\right),
\end{aligned}
$$


where the third equality comes from the fact that we are considering induction from the $\mathfrak{k}$-structure, so that the contribution of the roots of this Levi subalgebra should be removed from the character of $U(\mathfrak{g})$.

The above formula is reminiscent of Weyl-Kac character formula for integrable representations, but we have not yet found a reference for it in the literature. As we have explained in (4.1), this formula is an easy consequence of [KL94] and Lu89].

The equivalence in Theorem 4.1 is an equivalence of monoidal categories where the product on the left-hand side is the fusion tensor product $\hat{\otimes}$ and the product on the right-hand side is the tensor product coming from the Hopf algebra structure of $U_{q}(\mathfrak{g})$. Thus, in terms of affine Lie algebra representations, the Lusztig-Steinberg tensor product theorem says that

$$
\text { if } \lambda \in\left(\mathfrak{a}_{\mathbb{Z}}^{*}\right)^{+} \quad \text { and } \quad \lambda=\lambda_{0}+\ell \lambda_{1} \text { with } \lambda_{0} \in \Pi_{\ell},
$$

where $\Pi_{\ell}$ is as in (1.15). Then

$$
L\left((-\ell-h) \Lambda_{0}+\lambda\right) \cong L\left((-\ell-h) \Lambda_{0}+\lambda_{0}\right) \hat{\otimes} L\left((-\ell-h) \Lambda_{0}+\ell \lambda_{1}\right) .
$$

\section{ACKNOWLEDGMEnTs}

We thank all the institutions which have supported our work on this paper, particularly the University of Melbourne, the Australian Research Council, and ICERM (Institute for Computational and Experimental Research in Mathematics). We thank Kari Vilonen and Ting Xue for their generous support of a visit of Martina Lanini to the University of Melbourne.

\section{REFERENCES}

[BBF] Ben Brubaker, Daniel Bump, and Solomon Friedberg, Matrix coefficients and IwahoriHecke algebra modules, Adv. Math. 299 (2016), 247-271, DOI 10.1016/j.aim.2016.05.012. MR.3519469

[CS] W. Casselman and J. Shalika, The unramified principal series of p-adic groups. II. The Whittaker function, Compositio Math. 41 (1980), no. 2, 207-231. MR581582

[CP] Vyjayanthi Chari and Andrew Pressley, A guide to quantum groups, Cambridge University Press, Cambridge, 1994. MR.1300632

[FGV] E. Frenkel, D. Gaitsgory, and K. Vilonen, Whittaker patterns in the geometry of moduli spaces of bundles on curves, Ann. of Math. (2) 153 (2001), no. 3, 699-748, DOI 10.2307/2661366. MR 1836286

[GH] I. Grojnowski and M. Haiman, Affine Hecke algebras and positivity of LLT and Macdonald polynomials, 2007, available from http://math.berkeley.edu/ haiman

$[\mathrm{Gu}]$ Jérémie Guilhot, Admissible subsets and Littelmann paths in affine Kazhdan-Lusztig theory, Transform. Groups 23 (2018), no. 4, 915-938, DOI 10.1007/s00031-018-9495-4. MR.3869423

[HKP] Thomas J. Haines, Robert E. Kottwitz, and Amritanshu Prasad, Iwahori-Hecke algebras, J. Ramanujan Math. Soc. 25 (2010), no. 2, 113-145. MR2642451

[JM] Gordon James and Andrew Mathas, A q-analogue of the Jantzen-Schaper theorem, Proc. London Math. Soc. (3) 74 (1997), no. 2, 241-274, DOI 10.1112/S0024611597000099. MR.1425323

[Kac] Victor G. Kac, Infinite-dimensional Lie algebras, 3rd ed., Cambridge University Press, Cambridge, 1990. MR1104219

[KMS] M. Kashiwara, T. Miwa, and E. Stern, Decomposition of q-deformed Fock spaces, Selecta Math. (N.S.) 1 (1995), no. 4, 787-805, DOI 10.1007/BF01587910. MR.1383585

[KT95] Masaki Kashiwara and Toshiyuki Tanisaki, Kazhdan-Lusztig conjecture for affine Lie algebras with negative level, Duke Math. J. 77 (1995), no. 1, 21-62, DOI 10.1215/S00127094-95-07702-3. MR 1317626

[KL94] D. Kazhdan and G. Lusztig, Tensor structures arising from affine Lie algebras. IV, J. Amer. Math. Soc. 7 (1994), no. 2, 383-453, DOI 10.2307/2152763. MR.1239507 
[Knp] Friedrich Knop, On the Kazhdan-Lusztig basis of a spherical Hecke algebra, Represent. Theory 9 (2005), 417-425, DOI 10.1090/S1088-4165-05-00237-2. MR2142817

[LRS] M. Lanini, A. Ram, and P. Sobaje, Fock space model for decomposition numbers for quantum groups at roots of unity, to appear in Kyoto Math. J., arXiv:1612.03120.

[LLT] Alain Lascoux, Bernard Leclerc, and Jean-Yves Thibon, Ribbon tableaux, Hall-Littlewood functions, quantum affine algebras, and unipotent varieties, J. Math. Phys. 38 (1997), no. 2, 1041-1068, DOI 10.1063/1.531807. MR1434225

[LT] Bernard Leclerc and Jean-Yves Thibon, Littlewood-Richardson coefficients and KazhdanLusztig polynomials, Combinatorial methods in representation theory (Kyoto, 1998), Adv. Stud. Pure Math., vol. 28, Kinokuniya, Tokyo, 2000, pp. 155-220. MR1864481

[Lcy] Cédric Lecouvey, Parabolic Kazhdan-Lusztig polynomials, plethysm and generalized HallLittlewood functions for classical types, European J. Combin. 30 (2009), no. 1, 157-191, DOI 10.1016/j.ejc.2008.02.007. MR2460224

[Li] Peter Littelmann, Paths and root operators in representation theory, Ann. of Math. (2) 142 (1995), no. 3, 499-525, DOI 10.2307/2118553. MR.1356780

[Lu89] G. Lusztig, Modular representations and quantum groups, Classical groups and related topics (Beijing, 1987), Contemp. Math., vol. 82, Amer. Math. Soc., Providence, RI, 1989, pp. 59-77, DOI 10.1090/conm/082/982278. MR.982278

[Lu94] George Lusztig, Monodromic systems on affine flag manifolds, Proc. Roy. Soc. London Ser. A 445 (1994), no. 1923, 231-246, DOI 10.1098/rspa.1994.0058. MR1276910

[Lu95] George Lusztig, Errata: "Monodromic systems on affine flag manifolds" [Proc. Roy. Soc. London Ser. A 445 (1994), no. 1923, 231-246; MR1276910], Proc. Roy. Soc. London Ser. A 450 (1995), no. 1940, 731-732. MR2105507

[NR] Kendra Nelsen and Arun Ram, Kostka-Foulkes polynomials and Macdonald spherical functions, Surveys in combinatorics, 2003 (Bangor), London Math. Soc. Lecture Note Ser., vol. 307, Cambridge Univ. Press, Cambridge, 2003, pp. 325-370. MR2011741

[NP] B. C. Ngô and P. Polo, Résolutions de Demazure affines et formule de Casselman-Shalika géométrique (French, with English summary), J. Algebraic Geom. 10 (2001), no. 3, 515547. MR 1832331

[Ra] Arun Ram, Alcove walks, Hecke algebras, spherical functions, crystals and column strict tableaux, Pure Appl. Math. Q. 2 (2006), no. 4, Special Issue: In honor of Robert D. MacPherson., 963-1013, DOI 10.4310/PAMQ.2006.v2.n4.a4. MR2282411

[Sh] Peng Shan, Graded decomposition matrices of v-Schur algebras via Jantzen filtration, Represent. Theory 16 (2012), 212-269, DOI 10.1090/S1088-4165-2012-00416-2. MR2915315

School of Mathematics, University of Edinburgh, James Clerk Maxwell Building, EDINBURGH EH9 3FD UNited Kingdom

Email address: lanini@mat.uniroma2.it

Department of Mathematics and Statistics, University of Melbourne, Parkville ViC 3010 Australia

Email address: aram@unimelb.edu.au 